\title{
Non-Cancer Pain in the Adult Population: An Integrative Review
}

\author{
Alqahtani Jawhrah Mohammed \\ King Saud University, School of Nursing, Riyadh 11451, Saudi Arabia \\ Corresponding author: Alqahtani Jawhrah Mohammed; jawhrah.alqahtani@mymail.barry.edu
}

Received 26 November 2019;

Accepted 15 December 2019;

Published 20 December 2019

\begin{abstract}
Pain is a global phenomenon with significant physical, psychological, financial, and social impacts. The number of patients with pain has increased globally, due to the increase in the complexity of the pain and its classifications. Despite pain's high prevalence and economic impact, this phenomenon is not well investigated and understood. Understanding the concept of adult, non-cancer pain via the Specificity Theory and the Gate Control Theory of Pain is critical for professionals to expand the understanding of pain and ensure the best care. The purpose of this integrative review paper was to conduct a concept identification and analysis on pain through analyzing the existing literature. As the literature was reviewed, three major concepts emerged. Suffering, discomfort, and coping were the most frequent concepts that emerged, which is consistent with several studies of different populations, utilizing quantitative and qualitative methods. As pain concept is relevant to patient care and nursing practice, pain needs to be investigated by healthcare professionals and nursing research to provide a complete picture of the nature and pattern of pain in patients with chronic conditions such as genetic disorders, and in generating more knowledge about patient experiences. The importance of understanding and viewing pain via multiple lenses allows all to understand pain as multifaceted, complex, and interdisciplinary issues for which several of the causes are the social determinants of health.
\end{abstract}

Keywords: Non-cancerous pain, Chronic pain, Factors related to pain.

\section{Background}

Pain is a global phenomenon with significant physical, psychological, financial, and social impacts ${ }^{[15]}$. Pain includes distressing symptoms and is a common reason for individuals to seek health care, yet insufficient pain relief continues to be a challenge $^{[16]}$. Worldwide, the economic burden of pain on the health care system and patient life is drastic, as the annual cost of pain exceeds the cost of both cancer and heart diseases ${ }^{[11]}$. Due to the fact that providers still lack basic knowledge about what pain and its appropriate management are, pain remains a major global healthcare issue ${ }^{[16]}$. Thus, a clear understanding of the concept of non-cancerous pain can enhance care and improve treatment.

The definition of pain is based on McCaffery (1968), the International Association for the Study of Pain (IASP) (1979), and the Miller-Keane Encyclopedia \& Dictionary of Medicine, Nursing, and Allied Health (1997), are most frequently utilized across literature ${ }^{[15,19,14,20]}$. In 1968, McCaffery defined pain as whatever the patient experiencing it says it is, existing whenever the patient says it does, while the IASP (1979) said it is an unpleasant sensory and physical experience associated with actual or and potential damage that can be classified in terms of such damage ${ }^{[18,19,14]}$. Carroll- Johnson (1989), defined pain, in nursing diagnosis, as when "an individual experiences and reports the presence of severe discomfort or an uncomfortable sensation" ${ }^{[7]}$. The Miller-Keane Encyclopedia \& Dictionary of Medicine,
Nursing, and Allied Health (1997) defined pain as a feeling of distress or suffering, caused by stimulation of specialized nerve endings ${ }^{[20]}$. In 2012, the World Health Organization considered pain as tissue damage that can lead to unpleasant experiences ${ }^{[27]}$. The Institute of Medicine (2012) called it a subjective, complex phenomenon with physical, psychological, social, and spiritual processes associated with actual or potential tissue damage ${ }^{[13]}$. The current state of knowledge describes pain as a complex or multifaceted phenomenon which involves highly variable factors: failure to integrate physical, psychosocial, genetic, and behavioral factors that can lead to an incomplete understanding of pain ${ }^{[2]}$.

Despite pain's high prevalence and economic impact, this phenomenon is not well investigated and understood ${ }^{[2,15]}$. Defining the concept of pain is essential to the future of pain research and improves the quality of patient care.

\section{Purpose}

The purpose of this integrative review is to expand the understanding of the concept of an adult, non-cancer pain. The definition of pain and the common theoretical perspective of it are presented. Various medical, nursing, psychology, and allied health literature published are searched to identify a consensual definition of pain and its related concepts. Since concepts such as suffering, discomfort, and coping are commonly related to pain, it is important to describe their close relationship. A literature review of 
this concept and its implications for future research are also discussed.

\section{Theoretical Perspective}

Over the past decade, several theoretical frameworks have been established to explain the physiological basis of pain and understand the complex phenomenon of the pain process. The Specificity Theory and the Gate Control Theory of Pain (GCT) are two of the most influential theories of pain used to discuss this concept.

\section{Specificity Theory}

The Specificity Theory, developed by Schiff and Von Frey, proposed that pain is a sensory modality like vision or hearing or as an independent sensation with its own/ specialized central and peripheral sensory apparatus independent of touch, which responds to damage "pain impulses" and send signals via different neural pathways in the nervous system to target pain centers in the brain $^{[3]}$. This theory emphasizes that each sensory modality is encoded by specific pain receptors and the pain centers in the brain respond to each stimuli and process the signals to produce the experience of pain ${ }^{[6]}$. This asserts that pain involves physiological stimulus, as there is a fiber that leads a specific pain pathway directly to the sensory modality's region of the brain. The theory considers pain experiences as equivalent to peripheral injury ${ }^{[3]}$. This theory, therefore, suggests that a pathway-specific pain exists and the degree and intensity of damage determines the extent of pain experienced ${ }^{[3]}$.

\section{Gate Control Theory of Pain}

The Gate Control Theory (GCT), formulated by Melzack and Wall, proposes that pain is a function of the balance between the information traveling to the spinal cord via large and small nerve fibers ${ }^{[19]}$. The theory's emphasis that there is a neurological gate in the spinal cord that controls the transmission of pain signals by either allows or blocks pain signals from continuing to the brain ${ }^{[19]}$. It declares that individuals experience more pain when the "neurological gate" is open than when it is closed by activity in large nerve fibers ${ }^{[4,19]}$. The GCT explains that pain experience relies on the complex interaction of both the central and peripheral nervous system: it highlights the dynamic role of the brain in pain processes ${ }^{[4,19]}$; affirming that people bring their own understanding, experiences, memories, expectations, and culture to their own pain experience $^{[5,1]}$. It further asserts that pain is a multi-dimensional experience which must account for cognitive factors and psychological effects because it is the first theory to consider the psychological aspects of pain experience and perception in addition to the physiological basis of pain ${ }^{[4,11]}$. Thus, the GCT provides crucial information to reconciling the apparent differences with Specificity Theories and understanding pain more broadly.

Both theories have heuristic value in explaining pain concepts, mechanisms, perceptions, and experiences, and most importantly have a critical influence on pain research, treatment, and management ${ }^{[3,6]}$. Psychological and cognitive evaluation, and physical, emotional, cultural, and environmental factors must be considered to understand pain as a complete concept ${ }^{[12]}$. Since the development of Descartes' Specificity Theory and the GCT, there is an increasing awareness of the several factors that must account for pain experiences and pain care in the professional field ${ }^{[3]}$.

\section{Material and Method}

In this article use the method of meta-analysis of the basic literature and the results of previous research. A review of literature related to pain was conducted to obtain substantial information necessary for the understanding of this concept and its related concepts. A search of databases such as CINAHL, Cochrane Database, Ovid, ProQuest Nursing, PsycINFO, Allied Health Source, and MedLine, was conducted to locate common concepts and research studies discussion of this concept. Keywords used in this advanced search included: non-cancerous pain, chronic pain, persistent pain, adult patients, and factors related to pain. The review was limited to studies published in full-text English and within ten years of publication, from 2010 to 2019. All abstracts were reviewed to ensure transparency and relevance to the topic. Suffering, discomfort, and coping are the most common concepts that emerge, which is consistent with several studies of different populations, utilizing quantitative and qualitative methods.

\section{Discussion}

\section{The Concepts of Pain}

Although the concepts of pain and suffering are distinct, these concepts are often used interchangeably, and this is due to the high prevalence of chronic diseases, uncontrolled pain, and high rates of healthcare utilization ${ }^{[17,10]}$. Both concepts are closely related and have been recognized repeatedly in literature, especially in patients with chronic conditions such as sickle cell disease ${ }^{[10,22]}$. Many sources of suffering have been emphasized, including the pain itself, functional limitations, anxiety, depression, and social issues caused by the uncontrolled pain ${ }^{[10,17]}$. The amount of suffering has been found to be directly related to the severity and duration of pain and the number of physical and psychosocial consequences ${ }^{[23]}$. The evidence has related pain to suffering as a dimension and has suggested that suffering is complex as it involves individuals' perceptions, culture, beliefs, and interactions within their environment, in addition to physical pain, yet suitable treatment may help relieve suffering ${ }^{[17]}$. Suffering is also linked to pain behaviors: cognitive behavioral therapy can be employed to ease it $^{[9.10]}$. Professionals must include dimensions of suffering when assessing and treating a patient's pain.

Discomfort is usually related to pain ${ }^{[2,21]}$. Discomfort is characterized by an unpleasant feeling which can be physical or psychological and is recognized by self-report or observation ${ }^{[2,16]}$. Pain is one of the predominant causes for discomfort, yet not every discomfort can be attributed to pain ${ }^{[1,2]}$. Discomfort and pain are associated with reduction in quality of life $\mathrm{f}^{[21,22]}$. Evidence shows that individuals living with chronic pain commonly experience disturbed sleep and a reduction in physical and mental functioning: patients with chronic diseases such as cancer and genetic disorders are more likely to feel uncomfortable and develop psychological disorders such as depression when they cannot handle the pain ${ }^{[25,22]}$. Assessment of discomfort is vital for caring for patients with pain, so looking for sources of discomfort and treating them properly are essential components of pain care ${ }^{[2,5,7]}$.

Coping is a concept found in the literature, usually related to pain $^{[16,8,4]}$. Coping is a widely used term meaning dealing with stressful conditions; it is used when assessing and treating patients with pain ${ }^{[8]}$. Coping is viewed as the use of cognitive appraisal and behavioral actions and the use of available resources in order to achieve physiological and psychological adjustment ${ }^{[8]}$. Pain is a major stressor in the lives of patients with chronic disorders, and due to that, these patients are more likely to develop behavioral, psychological, and adjustment problems ${ }^{[27,28]}$. The effectiveness of coping strategies such as acupressure, cognitive distraction, and guided imagery is well documented ${ }^{[23,27,8]}$. These coping strategies help to diminish the negative effects of pain, reduce the 
consumption of pain medications, and increase daily activities ${ }^{[8,15]}$. Evidence shows that effective coping strategies predict adjustment beyond the individual's pain and illness, as in sickle cell populations, while negative coping responses are associated with more frequent pain, more significant pain, and psychological maladjustment ${ }^{[5]}$. Coping strategies are linked to an individual's physical and mental health; the ability to cope with pain is significantly important to help patients adjust to the stressor successfully and maintain their general well-being ${ }^{[16]}$.

\section{The Concepts of Pain Related to Suffering, Discomfort, and Coping}

Frankl and Saunders $(1962,1963)$ have found empirical support for a strong relationship between physical pain and psychosocial suffering (emotional distress) when physical pain and symptoms are controlled and alleviated; psychological pain is often lifted ${ }^{[9]}$. These old findings have been confirmed in a current quantitative cross-sectional study published in the International Journal of Psychiatry in Medicine of Edwards et., al (2014). Researchers have conducted this study to determine the influence of emotional reactions to pain as predictors of psychological distress in adult blacks patients with $\mathrm{SCD}^{[9]}$. Results reveals that there is a significant relationship between psychological pain and the experience of chronic pain in SCD patients ${ }^{[9]}$. It has been further discovered that psychosocial suffering is highly associated with SCD pain, as there is a greater negative emotional distress associated with $\mathrm{it}^{[9]}$. It highlights that the emotional reactions to pain have negatively affected various aspects of patients' lives and led to increased psychological pain, social isolation, mood changes, and poor self-care ${ }^{[9]}$. It has been determined that social and psychological support may help in enhancing not only psychological but also physical health-related functioning ${ }^{[9]}$. Understanding the meaning of pain and the nature of the physiology and psychology of pain comprehensively help providing insight into the unique and complex experience of it.

A cross-sectional study at an outpatient clinic in the large Urban Comprehensive Sickle Cell Center, was undertaken by Sanders, Labott, Molokie, Shelby, and Amp that looks at the relationship between pain, coping, and quality of life in adult patients with SCD, and to assess the influence of these factors on health service utilization ${ }^{[22]}$. The study has indicated that recurrent, painful, vaso-occlusive crisis is the primary reason for hospitalization and emergency room visits, with significant pain intensity ${ }^{[22]}$. Psychological coping and age have played a critical role in healthcare utilization, as the older patients have more outpatient visits to control pain, while the younger once have more emergency visits ${ }^{[22]}$. It concludes that there are differences between the age groups in terms of utilizing coping strategies; the younger group used heat, cold, or massage, while the older group used more passive coping (prayer and hoping) to cope with their pain ${ }^{[22]}$. It is highlighted that education about coping strategies helps to combat the negative implications of pain in the patient's $\operatorname{life}^{[22]}$. A multidimensional analysis of pain phenomena in populations with genetic and chronic disorders is needed to fully understand the nature and pattern of pain, its emotional aspects, and improved pain care.

The Cognitive-Behavioral Therapy (CBT), an adjunct to standard treatment, is utilized to increase comfort and offer psychological support that can aid in the control and treatment of chronic pain ${ }^{[12]}$. Hofmann, Asnaani, Vonk, Sawyer, and Fang (2012) have conducted a comprehensive literature review and utilized the findings of 269 meta-analysis studies to determine the actual effectiveness of cognitive-behavioral therapy in improving chronic conditions for individuals with a wide range of health problems (16 disorders), including chronic pain and distress due to general medical conditions ${ }^{[12]}$. Their findings, based on their respective reviews of literature, conclude that there is strong evidence to support the efficacy of CBT for treating or controlling several conditions and symptoms, including reducing pain, distress, anxiety, and anger, resulting in help for various individuals to cope with their situations effectively ${ }^{[12]}$. It clarifies that pain and other symptoms can be managed effectively via enhancing confidence and positive coping methods, as this type of intervention appears to minimize levels of psychological suffering, leading to pain reduction related to emotional and physical factors ${ }^{[12]}$. By including CBT within the treatment plan, discomfort and complications often experienced with pain are diminished, improving the patients' overall quality of life, and coping with their pain ${ }^{[12]}$. However, the researchers do note that further research is warranted ${ }^{[12]}$. Recognizing the negative alterations of chronic, non-cancer pain in patients' lives is significant to providers' clear understanding of the concept of pain and in administering of alternative approaches with traditional treatments such as CBT to provide adequate pain management.

Another literature review, conducted by Toye et al., (2013) for the purpose of exploring the chronic pain and investigating patients' experiences of chronic non-malignant pain, offers an understanding of how some aspects and challenges can be overcome $^{[27]}$. Results indicate that pain adversely impacts many dimensions of patients' lives, and the effects extend to their social lives ${ }^{[27]}$. Helping patients to move along with their pain and to play an active role in their care aids in self-empowerment and selfmanagement of their own pain ${ }^{[27]}$. It further emphasizes the importance of enhancing partnerships between patients and providers to ensure delivery of the comprehensive care needed to treat complex pain ${ }^{[27]}$. The outcomes further validate the need for a more holistic and individualized approach, with consideration of the potential difficulties of caring for these patients, uniqueness of pain experiences, and physical and psychological (illness uncertainty) effects of living with pain ${ }^{[26]}$. All are fundamental aspects of caring for patients ${ }^{[27]}$. The findings from this review suggest that the need for educational intervention for providers and nurses related to pain and its management lead to improved pain management outcomes for individuals ${ }^{[27]}$. It is therefore substantial for healthcare providers to have a clear understanding of the patients' experiences from the perspectives of those who live the experience to inform current clinical practice and pain care. Because examining patients' experiences with pain via a multidimensional lens can allow recognition of all possible causes and needs, resulting in understanding their pain experiences holistically and delivering optimal pain care ${ }^{[26]}$. Several studies taken from the patients' perspective with chronic pain have found that patients often feel anger, annoyance and frustration towards the healthcare team, as the latter rarely listen attentively to their concerns and understand their views ${ }^{[26]}$. Also, as pain is not visible, patients living with chronic pain often times do not receive as much attention from providers and proper pain treatment as other issues, resulting in mistrust between both and negative patientproviders experiences ${ }^{[26]}$.

\section{Conclusion}

Pain is one of the most common health problems that have adverse impacts on the quality of patient's life and the health care system. Without a clear conceptualization of pain, it can be quite difficult to evaluate patients' appropriately. Understanding this concept via 
the Specificity Theory and the GCT is critical for professionals to ensure the best care. Although pain is a widespread reason to seek medical care, support adaptation, social support, and comfort is a key to helping patients who experience frequent pain to cope with their disease.

\section{Recommendations}

Further pain research is needed to improve understanding and management of non-cancerous pain and to strengthen the quality of healthcare services and nursing practice. Although the physical aspects of pain often remain a predominant focus in research and practice, social, psychological, and spiritual determinants of pain must be taken into consideration as an essential part of pain management. Pain also needs to be addressed in terms of its impact on family members and caregivers of these patients, making it an essential component of the pain definition. Recommendations for change in pain evaluation and clinical practice are based on the best evidence that suggests integrating diverse specialties, along with patients, in managing and relieving patients' pain. Recommendations for the future advancement of current practice includes using proven non-pharmacological interventions in combination with current treatments that can enhance overall care. Although pain is a universal phenomenon, professionals still have inadequate knowledge and training in it, and integrated care is virtually absent. Ultimately, examining patients' experiences with pain via a multidimensional lens that allows recognition of all possible causes and needs, is critical. By doing this, nurse and other professionals are in a better position to understand pain experiences holistically and deliver optimal pain care.

\section{References}

[1] Apkarian, A. V., Baliki, M. N., \& Geha, P. Y. (2009). Towards a theory of chronic pain. Progress in Neurobiology, $\quad$ 87(2), 81-97. https://www.ncbi.nlm.nih.gov/pubmed/18952143

[2] Ashkenazy, S., \& Ganz, F. D. (2019). The differentiation between pain and discomfort: A concept analysis of discomfort. Pain Management Nursing, 20 (4), 303-398. https://www.ncbi.nlm.nih.gov/pubmed/31307870

[3] Aydede, M. (2006). A critical and quasi-historical essay on theories of pain. In Pain: New Essays on Its Nature and the Methodology of Its, 1-58. http://citeseerx.ist.psu.edu/viewdoc/summary?doi=10.1.1 .207 .8060

[4] Bonica, J. J. (1990). History of pain concepts and therapies and management pain. Mt Sinai J Medicine, 58 (3), 191-202.

https://www.ncbi.nlm.nih.gov/pubmed/1875956

[5] Booker, M. J., Blethyn, K. L., Wright, C. J., \& Greenfield, S. M. (2006). Pain management in sickle cell disease. Chronic Illness, 2(1), 39-50. https://journals.sagepub.com/doi/abs/10.1177/174239530 60020011101

[6] Brannon, L. \& Feist, J. (2000). Health psychology: An introduction to behaviour and health. London: Wadsworth, Thomson Learning. https://pdfs.semanticscholar.org/6948/1205e12eba41753 c3aefff9417ba714f4db7.pdf

[7] Carroll-Johnson, R. M. (Ed.). (1989). Classification of nursing diagnoses: Proceedings of the eight conference, North American nursing diagnosis association.
Lippincott Williams \& Wilkins. https://www.worldcat.org/title/classification-of-nursingdiagnoses-proceedings-of-the-eighth-conference-northamerican-nursing-diagnosis-association/oclc/19266643

[8] Dysvik, E., Natvig, G. K., Eikeland, O. J., \& Lindstrøm, T. C. (2005). Coping with chronic pain. International Journal of Nursing Studies, 42(3), 297-305. https://www.sciencedirect.com/science/article/pii/S00207 48904001154?via\%3Dihub

[9] Edwards, C. L., Killough, A., Wood, M., Doyle, T., Feliu, M., Barker, C. S., \& O'Garo, K. G. N. (2014). Emotional reactions to pain predict psychological distress in adult patients with Sickle Cell Disease (SCD). The International Journal of Psychiatry in Medicine, 47(1), 116.https://www.ncbi.nlm.nih.gov/pmc/articles/PMC4444 040/

[10] Encarnacao P, Oliveira CC, Martins T (2015) Pain and suffering intertwined concepts: Perspectives and challenges for nurses. Palliative Care Journal, 2 (1), 2231. https://journals.sagepub.com/home/pal

[11] Henschke, N., Kamper, S. J., \& Maher, C. G. (2015). The epidemiology and economic consequences of pain. In Mayo Clinic Proceedings, 90 (1), 139-147. https://www.sciencedirect.com/science/article/abs/pii/S0 025619614008659?via\%3Dihub

[12] Hofmann, S. G., Asnaani, A., Vonk, I. J., Sawyer, A. T., \& Fang, A. (2012). The efficacy of cognitive behavioral therapy: A review of meta-analyses. Cognitive Therapy and Research, 36(5), 427-440.https://www.ncbi.nlm. nih.gov/pmc/articles/PMC3584580/

[13] Institute of Medicine. (2012). Living well with chronic illness: A call for public health action. https://www.nap.edu/catalog/13272/living-well-withchronic-illness-a-call-for-public-health

[14] International Association for the Study of Pain. (1979). IASP Taxonomy. https://www.iasppain.org/Education/Content.aspx?ItemNumber=1698

[15] International Association for the Study of Pain. (2013). Unrelieved pain is a major global healthcare problem. http://www.iasp-pain.org/

[16] Jensen, M. P., \& Turk, D. C. (2014). Contributions of psychology to the understanding and treatment of people with chronic pain: Why it matters to all psychologists. American Psychologist, 69(2), 105. https://psycnet.apa.org/record/2014-04960-001

[17] Krikorian, A., Limonero, J. T., \& Maté, J. (2012). Suffering and distress at the end-of-life. PsychoOncology, 21(8), 799-808. https://onlinelibrary.wiley.com/doi/full/10.1002/pon.208 7

[18] McCaffery, M. (1968). Nursing practice theories related to cognition. Bodily Pain and Man-Environment Interactions. University of California, Los Angeles. https://www.worldcat.org/title/nursing-practice-theoriesrelated-to-cognition-bodily-pain-and-man-environmentinteractions/oclc/1185286

[19] Melzack, R., \& Wall, P. D. (1965). Pain mechanisms: A new theory. Science, 150 (3699), 971-979. https://pdfs.semanticscholar.org/38d2/be60471398c102c 148b998b093a779773e3a.pdf

[20] Miller, B. F., \& Keane, C. B. (1997). Encyclopedia and dictionary of medicine, nursing, and allied health (6th ed.) Saunders. Louis, MO: Mosby. 
https://www.elsevier.com/books/miller-keane-

encyclopedia-and-dictionary-of-medicine-nursing-andallied-health/miller-keane/978-0-7216-9791-8

[21] Samuelson, K. A. (2011). Adult intensive care patients' perception of endotracheal tube-related discomforts: A prospective evaluation. Heart \& Lung, 40(1), 49-55. https://www.sciencedirect.com/science/article/pii/S01479 56310000026

[22] Sanders, K., Labott, S., Molokie, R., Shelby, S., \&amp; DeSimone, J. (2010). Pain, coping and health care utilization in younger and older adults with sickle cell disease. Journal of Health Psychology, 15(1), 131-137. https://journals.sagepub.com/doi/abs/10.1177/135910530 9345554

[23] Spacek, A. (2006). Modern concepts of acute and chronic pain management. Biomedicine \& pharmacotherapy, 60 (7), 329-335. https://www.sciencedirect.com/science/article/pii/S07533 32206001016

[24] Saunders, C. (1963). The treatment of intractable pain in terminal cancer. Proceedings of the Royal Society of Medicine, $56(3)$, 195-197. https://www.ncbi.nlm.nih.gov/pmc/articles/PMC1897373

[25] Saunders, C. (1963). Distress in dying. British medical journal, $\quad 2 \quad$ (5359), 746. https://www.ncbi.nlm.nih.gov/pmc/articles/PMC1872872
[26] Snelgrove, S., Edwards, S., \& Liossi, C. (2013). A longitudinal study of patients' experiences of chronic low back pain using interpretative phenomenological analysis: Changes and consistencies. Psychology \& Health, 28(2), 121-138. https://www.ncbi.nlm.nih.gov/pubmed/22149060

[27] Toye, F., Seers, K., Allcock, N., Briggs, M., Carr, E., Andrews, J., \& Barker, K. (2013). Patients' experiences of chronic non-malignant musculoskeletal pain: A qualitative systematic review. Br J Gen Pract, 63(617), e829-e841. https://bjgp.org/content/63/617/e829.short

[28] World Health Organization. (2012). WHO Guidelines for the pharmacological and radiotherapeutic management of cancer pain in adults and adolescents. https://apps.who.int/iris/bitstream/handle/10665/279700/ 9789241550390-eng.pdf?ua=1

\section{Author Profile}

Alqahtani Jawhrah received a B.S. degree in Nursing from King Saud University in the Kingdom of Saudi Arabia, and received an M.S degree in Nursing Education from Roberts Wesleyan College in the United States of America respectively. Currently, she is completing her $\mathrm{PhD}$ in Nursing at Barry University in the state of Florida, the United States of America. 